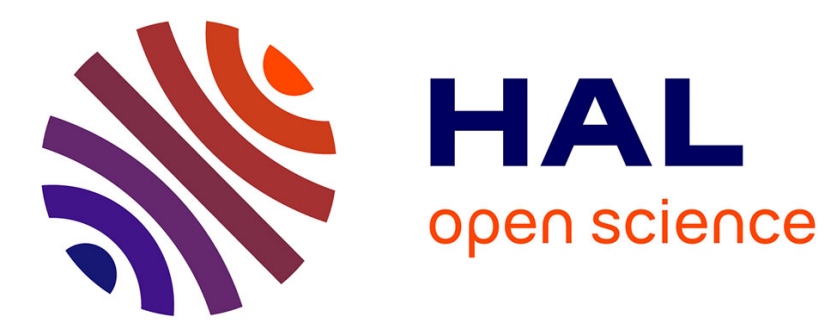

\title{
Local order theory of liquids : multipolar expansion
}

B. Quentrec, G. Bossis

\section{To cite this version:}

B. Quentrec, G. Bossis. Local order theory of liquids: multipolar expansion. Journal de Physique Lettres, 1980, 41 (5), pp.105-108. 10.1051/jphyslet:01980004105010500 . jpa-00231733

\section{HAL Id: jpa-00231733 https://hal.science/jpa-00231733}

Submitted on 1 Jan 1980

HAL is a multi-disciplinary open access archive for the deposit and dissemination of scientific research documents, whether they are published or not. The documents may come from teaching and research institutions in France or abroad, or from public or private research centers.
L'archive ouverte pluridisciplinaire HAL, est destinée au dépôt et à la diffusion de documents scientifiques de niveau recherche, publiés ou non, émanant des établissements d'enseignement et de recherche français ou étrangers, des laboratoires publics ou privés. 


\title{
Local order theory of liquids : multipolar expansion
}

\author{
B. Quentrec and G. Bossis \\ Laboratoire de Physique de la Matière Condensée (*), Parc Valrose, 06034 Nice Cedex, France
}

(Reçu le 26 septembre 1979, accepté le 7 janvier 1980)

\begin{abstract}
Résumé. - Nous montrons, utilisant un développement multipolaire de la densité d'énergie interne, qu'on a besoin de deux vecteurs et de trois tenseurs du deuxième ordre pour caractériser l'ordre local dans un liquide. Une interprétation simple de la signification physique de ces trois tenseurs est proposée.

Nous donnons les équations d'évolution de ces dernières variables et nous montrons leurs relations avec les variables hydrodynamiques habituelles.
\end{abstract}

\begin{abstract}
Using a multipolar expansion of the internal energy density we show that two vectors and three second rank tensors are necessary to characterize the local order in a liquid. A simple interpretation of the physical meaning of the three tensors is presented.

We give the equations of evolution of the latter variables and we show how they are connected to the usual hydrodynamic variables.
\end{abstract}

During the last decade the influence of the local order on the hydrodynamics of a liquid was emphasized by several authors $[1,2,3]$. In particular a number of studies have been concerned with the connection between the fine structure of the scattered light and the local order in a liquid [4], which so far has been described by the introduction of two tensorial quantities $[1,5,6]$. With these two tensors it is possible to explain a large number of experimental features. It is shown in reference [3] that these tensors called $\overleftrightarrow{\mathbf{G}}$ and $\overleftrightarrow{\mathbf{I}}$ can be introduced from angular momentum conservation. The tensor $\overleftrightarrow{\mathbf{G}}$ is related to the local order of the centre of gravity of the particles in a volume

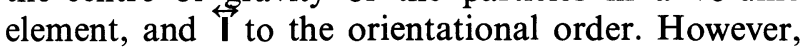
a systematic procedure describing local order was still to be developed. In this letter, we show that such a systematic procedure is obtained by a multipolar expansion of the internal energy which introduces two vectors and three tensors if the expansion is limited to the quadrupolar order. We give the equations of evolution of these vectors and tensors.

Consider a liquid system of rigid molecules. For the sake of simplicity we assume that the molecules are linear, so that they are characterized by the position of the centre of gravity $\mathbf{r}_{i}$ and the orientational vector $\mathbf{u}_{i}$. The vectors $\mathbf{u}_{i}$ are normalized. For a liquid of non rigid molecules, a polymer for instance,

$\left(^{*}\right)$ Laboratoire associé au C.N.R.S. $\mathrm{n}^{0} 190$. we could consider the polymer as a chain, the position of each rigid element of the chain being determined by the two vectors $\mathbf{r}_{i}$ and $\mathbf{u}_{i}$.

We consider the potential part of the energy density :

$$
e_{P}(\mathbf{r}, t)=\frac{1}{2} \sum_{i} \sum_{j \neq i} \Phi\left(\mathbf{r}_{i j}, \mathbf{u}_{i j}\right) U_{\xi}\left(\mathbf{r}_{i}-\mathbf{r}\right)
$$

The function $U_{\xi}(\mathbf{r})$ is equal to $1 / \xi^{3}$ if $\mathbf{r}$ is inside the cube of size $\xi$ centred at the origin and zero otherwhise. We call $\xi$ the correlation length of the local order. The function $\Phi\left(\mathbf{r}_{i j}, \mathbf{u}_{i j}\right)$ is the potential energy between molecule $i$ and molecule $j ; \mathbf{r}_{i j}=\mathbf{r}_{i}-\mathbf{r}_{j} ;$ and $\mathbf{u}_{i j}$ describes the relative orientation of molecules $i$ and $j$.

We recall [3] the definition of the hydrodynamic average of an operator $a(\mathbf{r}, t)$ :

$$
\langle a(\mathbf{r}, t)\rangle_{\mathrm{H}}=\frac{1}{\tau} \int_{t}^{t+\tau} a(\mathbf{r}, s) \mathrm{d} s
$$

where $\tau$ is a span of time that is short with respect to the relaxation time of the local order, but long with respect to the characteristic time of single particle motion. During the span of time $\tau$ it is possible to define a local thermodynamic equilibrium. Performing a hydrodynamic average, we eliminate the fast part of the behaviour of the molecules (vibrations and librations around the local equilibrium positions), and we keep only the slow part (relaxation of the local order). 
We now introduce the following definitions :

and

$$
n(\mathbf{r}, \mathbf{u}, t)=\left\langle\sum_{i} \delta\left(\mathbf{r}-\mathbf{r}_{i}(t)\right) \delta\left(\mathbf{u}-\mathbf{u}_{i}(t)\right)\right\rangle_{\mathbf{H}}
$$

$$
\Phi(\mathbf{r}, \mathbf{u}, t)=\frac{\left\langle\sum_{i} \sum_{i \neq j} \Phi\left(\mathbf{r}_{i j}(t), \mathbf{u}_{i j}(t)\right) \delta\left(\mathbf{r}-\mathbf{r}_{i}(t)\right) \delta\left(\mathbf{u}-\mathbf{u}_{i}(t)\right)\right\rangle_{\mathbf{H}}}{n(\mathbf{r}, \mathbf{u}, t)}
$$

when $n(\mathbf{r}, \mathbf{u}, t) \neq 0$. The functions $n(\mathbf{r}, \mathbf{u}, t)$ and $\Phi(\mathbf{r}, \mathbf{u}, t)$ are the hydrodynamic averaged density and potential respectively. With these definitions and from (1), we have

$$
\left\langle e_{\mathbf{P}}(\mathbf{r}, t)\right\rangle_{\mathbf{H}}=\frac{1}{2} \int n(\mathbf{x}, \mathbf{u}, t) \Phi(\mathbf{x}, \mathbf{u}, t) U_{\xi}(\mathbf{x}-\mathbf{r}) \mathrm{d} \mathbf{x} \mathrm{d} \mathbf{u} .
$$

In a solid $n(\mathbf{r}, \mathbf{u}, t)$ is independent of $t$ and is different from zero only for $\mathbf{r}$ around the lattice sites $\mathbf{r}_{p}$ and for $\mathbf{u}$ around the equilibrium positions $\mathbf{u}_{l}$. We call these small volumes in the (r, $\left.\mathbf{u}\right)$ space $V_{P l}$. The function $\Phi(\mathbf{r}, \mathbf{u}, t)$ is also independent of $t$ and its definition (4) holds for $(\mathbf{r}, \mathbf{u}) \in V_{P l}$. The average value $\Phi_{P l}$ of $\Phi(\mathbf{r}, \mathbf{u})$ in $V_{P l}$ is given by :

$$
\int_{V_{P l}} n(\mathbf{x}, \mathbf{u}) \Phi(\mathbf{x}, \mathbf{u}) \mathrm{d} \mathbf{x} \mathrm{d} \mathbf{u}=\Phi_{P l} \int_{V_{P l}} n(\mathbf{x}, \mathbf{u}) \mathrm{d} \mathbf{x} \mathrm{d} \mathbf{u} .
$$
that

By a smooth interpolation procedure we introduce a function $\Phi_{s}(\mathbf{r}, \mathbf{u})$ defined for each value of $(\mathbf{r}, \mathbf{u})$ such

$$
\Phi_{s}(\mathbf{r}, \mathbf{u})=\Phi_{P l} \quad \text { when } \quad(\mathbf{r}, \mathbf{u}) \in V_{P l}
$$

and

$$
\int n(\mathbf{x}, \mathbf{u}) \Phi(\mathbf{x}, \mathbf{u}) U_{\xi}(\mathbf{x}-\mathbf{r}) \mathrm{d} \mathbf{x} \mathrm{d} \mathbf{u}=\int n(\mathbf{x}, \mathbf{u}) \Phi_{s}(\mathbf{x}, \mathbf{u}) U_{\xi}(\mathbf{x}-\mathbf{r}) \mathrm{d} \mathbf{x} \mathrm{d} \mathbf{u}
$$

In a solid, except for very special cases, $V_{P l}$ is independent of $P$ and $l$. Therefore $\Phi_{s}(\mathbf{r}, \mathbf{u})$ is constant with respect to changes of $\mathbf{r}$ and $\mathbf{u}$.

In a dense liquid $n(\mathbf{r}, \mathbf{u}, t)$ varies very slowly during the span of time $\tau$ and the organization of the molecules in the box of size $\xi$ is like in a loose solid [3]. Using the above procedure, it is then possible to define a function $\Phi_{s}(\mathbf{r}, \mathbf{u}, t)$. Note that now the $\Phi_{P l}$ 's are no longer constant but vary slightly from one value of $(P, l)$ to another. Therefore $\Phi_{s}(\mathbf{r}, \mathbf{u}, t)$ is no longer a constant but a slowly varying function of $\mathbf{r}$ and $\mathbf{u}$ in the box of size $\xi$. It is then possible to perform a Taylor expansion of this function. Since the variation with respect to $\mathbf{u}$ is slow, any $\mathbf{u}$ can be taken as the origin of the expansion. We choose $\mathbf{u}_{z}$, the unit vector along the $z$ axis.

Using eqs. (5) and (8) we obtain :

$$
\begin{aligned}
\left\langle e_{P}(\mathbf{r}, t)\right\rangle_{\mathbf{H}}=\frac{1}{2} \rho(\mathbf{r}, t) \Phi_{s}\left(\mathbf{r}, \mathbf{u}_{z}, t\right)+\frac{1}{2} \mathbf{D}(\mathbf{r}, t) & . \nabla_{r} \Phi_{s}\left(\mathbf{r}, \mathbf{u}_{z}, t\right)+ \\
& +\frac{1}{2} \mathbf{m}(\mathbf{r}, t) \cdot \nabla_{u} \Phi_{s}\left(\mathbf{r}, \mathbf{u}_{z}, t\right)+\frac{1}{4} \overleftrightarrow{\mathbf{G}}(\mathbf{r}, t): \nabla_{r} \nabla_{r} \Phi_{s}\left(\mathbf{r}, \mathbf{u}_{z}, t\right) \\
& \left.+\frac{1}{4} \stackrel{\mathbf{l}}{\mathbf{r}}, t\right): \nabla_{u} \nabla_{u} \Phi_{s}\left(\mathbf{r}, \mathbf{u}_{z}, t\right)+\frac{1}{4} \stackrel{\mathbf{J}}{(}(\mathbf{r}, t): \nabla_{r} \nabla_{u} \Phi_{s}\left(\mathbf{r}, \mathbf{u}_{z}, t\right)+\cdots
\end{aligned}
$$

where

$$
\begin{aligned}
\rho(\mathbf{r}, t) & =\int n(\mathbf{x}, \mathbf{u}, t) U_{\xi}(\mathbf{x}-\mathbf{r}) \mathrm{d} \mathbf{x} \mathrm{d} \mathbf{u} \\
\mathbf{D}(\mathbf{r}, t) & =\int(\mathbf{x}-\mathbf{r}) n(\mathbf{x}, \mathbf{u}, t) U_{\xi}(\mathbf{x}-\mathbf{r}) \mathrm{d} \mathbf{x} \mathrm{d} \mathbf{u} \\
\mathbf{m}(\mathbf{r}, t) & =\int\left(\mathbf{u}-\mathbf{u}_{z}\right) n(\mathbf{x}, \mathbf{u}, t) U_{\xi}(\mathbf{x}-\mathbf{r}) \mathrm{d} \mathbf{x} \mathrm{d} \mathbf{u} \\
\overleftrightarrow{\mathbf{G}}(\mathbf{r}, t) & =\int(\mathbf{x}-\mathbf{r})(\mathbf{x}-\mathbf{r}) n(\mathbf{x}, \mathbf{u}, t) U_{\xi}(\mathbf{x}-\mathbf{r}) \mathrm{d} \mathbf{x} \mathrm{d} \mathbf{u} \\
\overleftrightarrow{\mathbf{I}}(\mathbf{r}, t) & =\int\left(\mathbf{u}-\mathbf{u}_{z}\right)\left(\mathbf{u}-\mathbf{u}_{z}\right) n(\mathbf{x}, \mathbf{u}, t) U_{\xi}(\mathbf{x}-\mathbf{r}) \mathrm{d} \mathbf{x} \mathrm{d} \mathbf{u} \\
\overleftrightarrow{\mathbf{J}}(\mathbf{r}, t) & =\int(\mathbf{x}-\mathbf{r})\left(\mathbf{u}-\mathbf{u}_{z}\right) n(\mathbf{x}, \mathbf{u}, t) U_{\xi}(\mathbf{x}-\mathbf{r}) \mathrm{d} \mathbf{x} \mathrm{d} \mathbf{u}
\end{aligned}
$$


The scalar $\rho$ is the number density. $\mathbf{m}$ is the vector of polarization and plays an important role in polar fluids [7] where the Fourier transform of

$$
c(t)=\int\langle\mathbf{m}(0,0) \cdot \mathbf{m}(\mathbf{r}, t)\rangle \mathrm{d} \mathbf{r}
$$

is related to the dielectric constant $\varepsilon(\omega)$. The vector D does not couple with an electromagnetic field in a neutral liquid, but will be related to $\varepsilon(\omega)$ in a molten salt or an electrolyte.

The tensors $\overleftrightarrow{\mathbf{G}}$ and $\overleftrightarrow{\mathbf{T}}$ are the quantities introduced in an earlier study [3] based on angular momentum conservation. The important feature of the present work is that the multipolar expansion introduces a third tensor $\overleftrightarrow{\mathbf{J}}$ which is independent of $\overleftrightarrow{\mathbf{G}}$ and $\overleftrightarrow{\mathbf{I}}$. So $\overleftrightarrow{\mathbf{J}}$ is not related to mode coupling arising from the effects

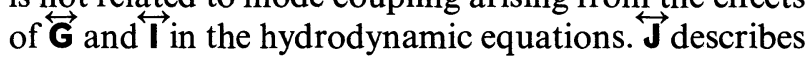
the correlation between centre of gravity and orientational order which can be viewed as a gearing effect between entangled molecules in a dense fluid (see Fig. 1). So $\overleftrightarrow{J}$ should be an important quantity in dense fluids of non-spherical non-symmetric top molecules like highly viscous liquids. Note also that $\overleftrightarrow{\mathbf{G}}$ and $\overleftrightarrow{\mathbf{I}}$ are invariant under orientation reversal $\left(\mathbf{u}_{i} \rightarrow-\mathbf{u}_{i}\right)$ whereas $\overleftrightarrow{J}$ is not.

The quantities $\nabla_{r} \Phi_{s}\left(\mathbf{r}, \mathbf{u}_{z}, t\right)$ and $\nabla_{u} \Phi_{s}\left(\mathbf{r}, \mathbf{u}_{z}, t\right)$ are respectively the averaged force and the averaged torque acting in $\mathbf{r}$ in the volume element dr during the span of time $\tau$ which modify the local order. By definition of the hydrodynamic average the variation of the local order is very slow during time $\tau$. Therefore $\nabla_{r} \Phi_{s}$ and $\nabla_{u} \Phi_{s}$ are very small and we shall neglect them hereafter.

We now present the equations of evolution for $\mathbf{m}(\mathbf{r}, t)$ and $\mathbf{D}(\mathbf{r}, t)$. From (3) and (10) we redefine $\mathbf{m}$ by subtracting the trivial part $\rho \mathbf{u}_{z}$

$$
\mathbf{m}(\mathbf{r}, t)=\left\langle\sum_{i} \mathbf{u}_{i}(t) U_{\xi}\left(\mathbf{r}_{i}-\mathbf{r}\right)\right\rangle_{\mathbf{H}}
$$

(1)

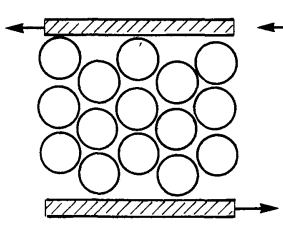

(2)

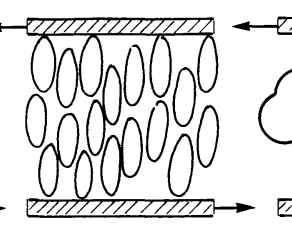

(3)

Fig. 1. - Representation of three typical local order structures. On each of these local order structure we apply a shear stress characterized by two arrows on each sketch : 1 . The local order is described by the $\overleftrightarrow{\mathbf{G}}$ tensor. The shear stress induces a variation of this tensor. 2. The local order is described by the tensors $\overleftrightarrow{\mathbf{G}}$

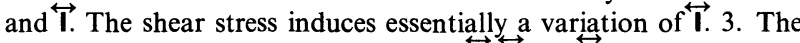

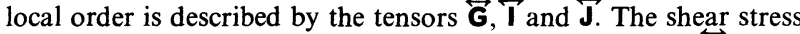
induces a variation of the three tensors, particularly of the $\overleftrightarrow{\mathbf{J}}$ tensor via a gearing effect. which by space Fourier transformation yields

$$
\begin{aligned}
\dot{\mathbf{m}}(\mathbf{k}, t)=\left\langle\sum_{i} \dot{\mathbf{u}}_{i} \mathrm{e}^{i \mathbf{k} \cdot \mathbf{r}_{i}} U_{\xi}(\mathbf{k})+\right. \\
\left.+i \mathbf{k} \cdot \sum_{i} \mathbf{v}_{i} \mathbf{u}_{i} \mathrm{e}^{i \mathbf{k} . \mathbf{r}_{i}} U_{\xi}(k)\right\rangle_{,}
\end{aligned}
$$

where $\mathbf{v}_{i}$ is the velocity of molecule $i$. Transforming back into $\mathbf{r}$ space we obtain

$$
\begin{aligned}
\dot{\mathbf{m}}(\mathbf{r}, t)=\left\langle\sum_{i} \dot{\mathbf{u}}_{i} U_{\xi}\left(\mathbf{r}_{i}-\mathbf{r}\right)-\right. \\
\left.\quad-\nabla \cdot\left(\sum_{i} \mathbf{v}_{i} \mathbf{u}_{i} U_{\xi}\left(\mathbf{r}_{i}-\mathbf{r}\right)\right)\right\rangle_{\mathbf{H}}
\end{aligned}
$$

with

$$
\dot{\mathbf{u}}_{i}=\mathbf{\Omega}_{i} \wedge \mathbf{u}_{i}
$$

where $\boldsymbol{\Omega}_{i}$ is the angular velocity of molecule $i$. Using expressions (4.1) and (4.7) of reference [3] and performing the hydrodynamic average of the right hand side of (13), we find

$$
\frac{\mathrm{d}}{\mathrm{d} t} \mathbf{m}=\mathbf{\Omega} \wedge \mathbf{m}-\frac{1}{\rho} \nabla \cdot(\boldsymbol{\omega} \wedge \overleftrightarrow{\mathrm{J}})
$$

where we have taken, as we do in the remainder the density per unit mass, e.g., $\mathbf{m}$ means

$$
\left\langle\sum_{i} \mathbf{u}_{i} U_{\xi}\left(\mathbf{r}_{i}-\mathbf{r}\right)\right\rangle_{\mathbf{H}} / \rho(\mathbf{r}, t) .
$$

The bulk angular velocity $\boldsymbol{\omega}$, the librational density $\boldsymbol{\Omega}$, and the external product $\boldsymbol{\omega} \wedge \overleftrightarrow{\mathrm{J}}$ are defined in reference [3]. The time derivative of $\mathbf{m}$ along a flowline is given by :

$$
\frac{\mathrm{d}}{\mathrm{d} t} \mathbf{m}=\dot{\mathbf{m}}+(\mathbf{v} \cdot \nabla) \mathbf{m} .
$$

Following a similar procedure, we gei for $\mathbf{D}$

$$
\frac{\mathrm{d}}{\mathrm{d} t} \mathbf{D}=-\frac{1}{\rho} \nabla \cdot(\omega \wedge \overleftrightarrow{\mathbf{G}})
$$

Eq. (15) will be useful for the computation of the dielectric constant $\varepsilon(\omega)$ of a polar fluid.

To obtain the equations of evolution of the tensors $\overleftrightarrow{\mathbf{G}}, \overleftrightarrow{\mathrm{I}}$ and $\overleftrightarrow{\mathrm{J}}$ we follow the same method as in reference [3]. We first write the variation of the internal energy which with (9) and without the dipolar terms reads

$$
\begin{aligned}
& \mathrm{d} e_{i}=T \mathrm{~d} S-P \mathrm{~d} V+\boldsymbol{\omega} \cdot \mathrm{d} \mathbf{M}+ \\
& \quad+\mathbf{\Omega} \cdot \mathrm{d} \mathbf{S}+\overleftrightarrow{\mathbf{F}}_{\mathbf{I}}: \overleftrightarrow{\mathrm{I}}+\overleftrightarrow{\mathbf{F}}_{\mathbf{G}}: \mathrm{d} \overleftrightarrow{\mathbf{G}}+\overleftrightarrow{\mathbf{F}_{\mathbf{J}}}: \mathrm{d} \overleftrightarrow{\mathbf{J}}+\ldots
\end{aligned}
$$

The difference between eq. (17) and eq. (5.3) of reference [3] is the presence of the term $\overleftrightarrow{\mathrm{F}}_{\mathbf{J}}: \mathrm{d} \overleftrightarrow{\mathrm{J}}$. Following the procedure described in [3], we obtain 
the same set of equations, except the eq. (5.10) of [3] which now, after linearization, becomes :

$$
\begin{aligned}
& \overleftrightarrow{\pi^{s}}=-2 \alpha_{11}(\nabla \mathbf{V})^{s}-2 \alpha_{12} \dot{\mathbf{G}^{s}}-2 \alpha_{13} \dot{\overrightarrow{\mathrm{I}^{s}}}-2 \alpha_{14} \dot{\overrightarrow{\mathrm{J}^{s}}} \\
& \overleftrightarrow{\mathbf{F}_{\mathbf{G}}^{s}}=-2 \alpha_{12}(\nabla \mathbf{V})^{s}-2 \alpha_{22} \stackrel{\dot{\mathbf{G}^{s}}}{{ }^{s}}-2 \alpha_{23} \dot{\mathrm{I}^{s}}-2 \alpha_{24} \dot{\mathrm{J}^{s}}
\end{aligned}
$$

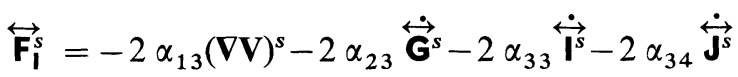

$$
\begin{aligned}
& \overleftrightarrow{\mathbf{F}_{\mathbf{J}}^{s}}=-2 \alpha_{14}(\mathbf{V V})^{s}-2 \alpha_{24} \overleftrightarrow{\mathbf{G}}^{s}-2 \alpha_{34} \stackrel{\dot{\mathbf{I}^{s}}}{{ }^{\prime}}-2 \alpha_{44} \dot{\mathbf{J}^{s}}
\end{aligned}
$$

and eq. (5.14) of [3] becomes

$$
\begin{aligned}
\pi_{t} & =-3 \varepsilon_{11} \nabla \cdot \mathbf{V}-3 \varepsilon_{12} \dot{G}_{t}-3 \varepsilon_{14} \dot{J}_{t} \\
\left(F_{G}\right)_{t} & =-3 \varepsilon_{124} \nabla \cdot \mathbf{V}-3 \varepsilon_{22} \dot{G}_{t}-3 \varepsilon_{24} \dot{J}_{t} \\
\left(F_{J}\right)_{t} & =-3 \varepsilon_{14} \nabla \cdot \mathbf{V}-3 \varepsilon_{24} \dot{G}_{t}-3 \varepsilon_{44} \dot{J}_{t}
\end{aligned}
$$

where $\overleftrightarrow{\pi^{s}}$ and $\pi_{t}$ are respectively the traceless part and the trace of the stress tensor. In eq. (15) we restrict the multipolar expansion to second order. Because of Curie's principle, in an isotropic fluid, coupling can exist only between tensors of the same order. The highest order tensor which appears in classical hydrodynamics is the stress tensor $\stackrel{\pi}{\pi}$. If the multipolar expansion is performed up to an order higher than two we can find contracted tensors of order two. For instance there are tensors of order four, which, after contraction reduce to tensors of order two which couple with the stress tensor. However as mentioned previously, since the variation of the local order is small over the distance $\xi$, the multipolar expansion must converge quickly. Therefore eq. (18) is certainly a good approximation since it amounts to neglect terms of order four with respect to terms of second order.

We now come back to the comparison between the earlier study [3] and the present work. In reference [3] it is shown that the tensors $\overleftrightarrow{\mathbf{G}}$ and $\overleftrightarrow{\mathbf{T}}$ are two slow variables which describe the local order. Up to equation (5.2) the results are a logical deduction from the introduction of angular momentum conservation. But eq. (5.3) which gives the variation of the internal energy is phenomenological. This equation takes into account the fast single particle motion with the terms $T \mathrm{~d} S-P \mathrm{~d} V$, and also the slow variation of the local order with the terms $\overleftrightarrow{\mathbf{F}_{\mathbf{I}}: \overleftrightarrow{d} \mathbf{I}}+\overleftrightarrow{\mathbf{F}_{\mathbf{G}}}:$ d $\overleftrightarrow{\mathbf{G}}$. But there

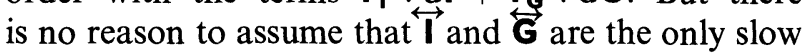
variables describing the local order. The advantage of the present work is to introduce a systematic procedure which gives all the variables related to the local order. Eq. (17) of the present work is no more phenomenological but the result of a multipolar expansion which introduces not only the tensors $\overleftrightarrow{\mathbf{G}}$

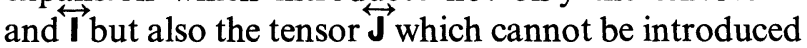
from angular momentum conservation.

Applications of the theory developed in the present work will be given in two forthcoming papers [8, 9] where it is shown that the introduction of the tensor $\overleftrightarrow{\mathbf{J}}$ is necessary to interpret ultrasonic and light scattering experiments in viscous fluids.

Acknowledgments. - One of the author (B. Q.) is indebted to C. Brot and G. Durand for a discussion where the importance was raised to introduce a third tensor. The authors are grateful to J. P. Boon for a critical review of the manuscript.

\section{References}

[1] Volterra, V., Phys. Rev. 180 (1969) 156.

[2] De Gennes, P. G., Mol. Cryst. Liq. Cryst. 12 (1971) 193.

[3] Quentrec, B., J. Physique 37 (1976) 1255.

[4] See for instance for a review Searby, G., Bezot, P. and Srxou, P., Faraday Discuss. Chem. Soc. 11 (1977) 63.
[5] Vaucamps, C., Chabrat, J. P., Letamendia, L., Nouchi, G. and Rouch, J., J. Physique 37 (1976) 1197.

[6] Quentrec, B., Phys. Rev. A 15 (1977) 1304.

[7] Bossis, G. and Quentrec, B., Mol. Phys. 32 (1976) 591.

[8] Eldin, G., Laheurte, J. P., Quentrec, B., to appear in J. Physique.

[9] Bezot, P., Hesse-Bezot, C., Quentrec, B., to appear. 